\title{
OS PROBLEMAS DE GESTÃO DO SUS DECORREM TAMBÉM DA CRISE CRÔNICA DE FINANCIAMENTO?
}

DO THE SUS MANAGEMENT PROBLEMS ALSO DERIVE FROM THE CHRONIC FUNDING CRISIS?

Carlos Octávio Ocké-Reis ${ }^{1}$

Resumo Como os formuladores de política podem lidar com a aspiração de produzir um sistema de saúde universal, em um contexto de restrição fiscal do Estado? Parece haver uma contradição entre o modelo redistributivo pressuposto na Constituição brasileira e o nível de gasto público em saúde. Neste quadro, o aumento de recursos financeiros é uma precondição para negarmos o SUS da 'não-universalidade' e da 'não-descentralização', para que ele não negue si mesmo enquanto direito social. Tal como o modelo de descentralização preconizado pelos ideólogos do SUS, que sofreu com a escassez de recursos, boa parte dos problemas de gestão decorre da crise crônica de financiamento, e a adoção de medidas de eficiência não pode servir de base para se cortar o nível de recursos financeiros ou organizacionais do SUS. Pelo contrário: a melhoria da eficiência pode, na realidade, significar e exigir o aumento dos gastos.

Palavras-chave saúde; gastos do governo e saúde;

Abstract How can those who formulate policies deal with the aspiration of producing a universal health context in a context of fiscal restrictions of the State? There seems to be a contradiction between the redistributive model the Brazilian Constitution predicates and the level of public expenditure with health. In this scenario, increasing financial resources is a precondition in order to deny the 'non-universal' and 'non-decentralized' SUS, so it will not deny itself as a social right. Such as the model of decentralization professed by SUS ideologues, a system that suffered from the scarcity of resources, most of the management problems result from the chronic funding crisis, and adopting efficiency measures cannot serve as the base to cut the SUS' level of financial or organizational resources. On the contrary: improved efficiency can, in fact, mean and require increased spending. Keywords health; government expenditures and health; efficiency. eficiência. 


\section{Introdução}

Concordamos com a tese central da professora Luciana Lima, quando afirma que "o modelo de federalismo fiscal, adotado sob os imperativos da política de estabilização econômica e contenção dos gastos em saúde, acabou gerando constrangimentos e desequilíbrios federativos e não favoreceu a redistribuição de recursos a favor das desigualdades nas condições de financiamento em saúde dos estados e municípios brasileiros".

Da mesma forma, temos acordo quando ela diz que "no contexto de restrição fiscal, o modelo de descentralização implementado no sistema de saúde serviu mais ao propósito de retração da União e de contenção de despesas do que de sua expansão, como gostariam seus idealizadores". A autora articula, corretamente, os problemas da descentralização com a crise crônica de financiamento, lançando mão de um conjunto de argumentos que nos ajudam a compreender inclusive o percurso desta crise do Sistema Único de Saúde (SUS), hoje, acentuada, com o fim da Contribuição Provisória sobre Movimentação Financeira (CPMF) no Senado Federal, em fins de 2007.

Nossa contribuição segue uma postura análoga a da professora, mas em uma direção complementar ao seu minucioso e consistente artigo: defender que boa parte dos problemas de gestão do SUS é decorrente da crise crônica de financiamento do SUS, aquela mesma que afetou os objetivos últimos da descentralização. Parece, assim, que a questão do financiamento continua sendo uma dimensão-chave para negarmos o SUS da 'não-universalidade' e da 'não-descentralização', para que ele não negue si mesmo enquanto direito social.

\section{Considerações sobre os problemas de gestão do SUS}

A Constituição de 1988 previu um modelo de financiamento adequado para garantir a cobertura universal? A partir da criação do Orçamento de Seguridade Social (OSS) esperava-se, ao menos, por meio do alargamento e diversificação da base de financiamento, superar a fragilidade do modelo prévio de seguro social - financiado com base na folha de salários. A necessidade de superar o gargalo do financiamento público do setor público, entretanto, foi interditada no seu nascedouro com o desmonte do OSS, dado que os $30 \%$ indicados nas disposições transitórias da Constituição foram derrubados, anunciando uma longa crise crônica de financiamento do SUS (Marques e Mendes, 1999). Nesse quadro, refém da dívida pública, a política fiscalista do governo (Castro et al., 2008) inibiu acentuadamente o nível do gasto público em saúde no Brasil, dificultando que o SUS assegure o 
acesso universal e integral, mas também supere seus problemas de gestão. Mas se a gestão do SUS pode ser melhorada, este argumento não pode ser usado como mote para a adoção de um controle indiscriminado de custos.

Este ponto de vista pode ser sustentado a partir da discussão do próprio conceito de eficiência. Eficiência pode ser entendida como a relação entre o valor dos recursos consumidos e os resultados obtidos. Logo, as medidas de eficiência podem mensurar quanto poderia ser produzido com os recursos existentes ou quais recursos deveriam ter sido consumidos para atingir determinado resultado. Tais avaliações são importantes na área da assistência à saúde, onde a eficiência é mensurada pela eficácia do tratamento e não por um simples mecanismo de mercado (Marinho e Façanha, 2001).

O Banco Mundial acredita que "aumentar a eficiência e efetividade no uso dos recursos de saúde para conter o aumento dos custos talvez seja o maior desafio posto para o sistema de saúde brasileiro" (Banco Mundial 2007). Sem dúvida, é necessário melhorar a eficiência do SUS; é necessário aumentar tanto os gastos sociais quanto garantir uma alocação eficiente (Mussi e Afonso, 2008). Afinal, quem argumentaria contra a introdução de um programa governamental ou serviço hospitalar mais barato, abrangente e eficaz?

Contudo, o que está por detrás desta tese do Banco Mundial? Identifica-se de forma pouco criteriosa o conceito de eficiência com medidas de contenção de custo, sem responder se a suposta ineficiência do SUS pode ter sido causada pelos escassos recursos públicos de custeio e investimento aplicados no sistema (Marinho, 2004). Na mesma linha, alguns analistas acreditam que o SUS já gasta o suficiente e que agora bastaria otimizar o gasto, por exemplo, aumentando a taxa de ocupação de leitos ou reduzindo os gastos hospitalares - por meio da expansão e melhoria da medicina preventiva e os serviços ambulatoriais (Pinheiro, 2008). Se otimizar o gasto é desejável e se a adoção de tais medidas são meritórias da ótica da organização do sistema e da perspectiva da qualificação da atenção médica, seu êxito pode prescindir - ao contrário - da ampliação dos recursos financeiros. Além do mais, no mundo dos negócios ou da administração pública, poderá sempre haver um certo nível de ineficiência extrapolada pelas idiossincrasias gerenciais, imprecisão das metas ou alvos organizacionais (Marinho e Façanha 2001).

Desse modo, a indagação correta a ser feita é: a contenção dos custos de maneira indiscriminada não poderia agravar os problemas de gestão do SUS e o próprio combate da alegada ineficiência sistêmica? Na verdade, o incremento da eficiência não deve ser tomado como desculpa para cortar recursos financeiros ou organizacionais do SUS, bem como as filas em um sistema universal de saúde não podem servir para restringir o acesso. A rigor, considerando o custo de oportunidade relativo à alocação de recursos para a 
saúde (considerando sua destinação a outros setores sociais), a melhor prática se pareceria com aquela ação racional realizada para 'valorizar o dinheiro' na execução dos serviços médico-hospitalares, prestados para garantir e melhorar as condições de saúde da população (melhor alocação, incentivos contratuais, meio-ambiente e tecnológico adequado, incremento da produtividade, corte de desperdícios, combate à corrupção etc.). Resumindo: a adoção de medidas de eficiência capazes de levar a melhores práticas não pode servir de base para se cortar o nível de recursos financeiros ou organizacionais do SUS; pelo contrário, a melhoria da eficiência pode, na realidade, exigir o aumento dos gastos (Marinho e Façanha, 2001).

Se parece aceitável reivindicar mais recursos para o SUS, na perspectiva inclusive de melhorar a gestão, seu crescimento, por meio da ampliação dos gastos públicos, tem meios limitados, e desse modo o governo não pode fechar os olhos diante da tendência de custos crescentes da assistência médica (Remler et al., 2005). Ainda assim, a proposta de uma nova fonte de financiamento estável e abrangente, defendida por amplos setores da sociedade e das instituições de Estado (Ipea, 2008), parece um caminho viável para atenuar a contradição entre o modelo redistributivo descrito na Constituição do Brasil e o nível de gasto público no Brasil.

\section{O subfinanciamento do SUS: uma abordagem internacional}

A análise dos gastos em saúde vem, crescentemente, atraindo economistas e cientistas políticos (Marmor; Okma; Latham, 2006; Delfim Netto, 2006). Se olharmos estudos comparativos que investigam diferentes sistemas de saúde, poderemos observar longas descrições de suas características; outros buscam analisar um conjunto de países para explicar sua história, aspectos comuns e diversidades, prescrevendo um conjunto de políticas a partir das experiências nacionais (Conill 2006; Marmor, 2001).

Não pretendemos fazer uma descrição detalhada, tampouco refletir sobre possíveis lições do caso brasileiro, principalmente em relação ao nível ótimo de financiamento do SUS. Fizemos tão-somente uma seleção ad hoc de países da América Latina e da Organização para Cooperação e Desenvolvimento Econômico (OCDE) e depois comparamos seus resultados com os do Brasil.

A Tabela 1 mostra que 7,6\% do PIB brasileiro é gasto em saúde, um pouco acima da média dos países latino-americanos, mas abaixo da Argentina. A proporção do gasto público com saúde em comparação ao gasto total é mais baixa do que a média de $54,4 \%$ da América Latina e inferior à média observada na OCDE. Cabe observar, entretanto, que em termos relativos o percentual do gasto público brasileiro (45,3\%) é semelhante ao dos EUA 
$(44,6 \%)$. Em termos per capita, o Brasil gasta US\$ 597, pouco abaixo da média dos países latino-americanos (US\$ 622) e cerca de cinco vezes menos que a média da OCDE.

Tabela 1

\begin{tabular}{lccc}
\hline \multicolumn{1}{l}{ Indicadores de gastos em saúde - 2003} & & \\
\hline Países & $\%$ PIB & \% Público & Per capita (PPP) US\$ \\
\hline Brasil & 7,6 & 45,3 & 597 \\
Argentina & 8,9 & 48,6 & 1,067 \\
Chile & 6,1 & 48,8 & 707 \\
Colômbia & 7,6 & 84,1 & 522 \\
México & 6,2 & 46,4 & 582 \\
Venezuela & 4,5 & 44,3 & 231 \\
Média América Latina & 6,7 & 54,4 & 622 \\
\hline Alemanha & 11,1 & 78,2 & 3.001 \\
Canadá & 9,9 & 69,9 & 2.989 \\
Holanda & 9,8 & 62,4 & 2.987 \\
Reino Unido & 8,0 & 85,7 & 2.389 \\
Estados Unidos & 15,2 & 44,6 & 5.711 \\
Média OCDE & 10,8 & 68,2 & 3.415 \\
\hline
\end{tabular}

Fonte: World Health Statistics 2006; The World Health Report 2006; World Bank 2006 (World Development indicators). Notas: Elaboração Ipea; PPP - Purchasing Power Prity (paridade o poder de compra).

A Tabela 2 (ver na página seguinte) mostra que a proporção de médicos no Brasil (2,1 por mil habitantes) é maior do que a média da América Latina e próxima à média da $\operatorname{OCDE}(2,6)$. A taxa de enfermeiros no Brasil é quase a metade da média da América Latina e vinte vezes menor do que a média OCDE, apesar dos custos mais baixos de contratação e do impacto positivo do seu trabalho sobre a efetividade dos serviços de saúde (Fagin, 1990). Paralelamente, existem 2,6 leitos hospitalares por mil habitantes no Brasil, taxa mais alta do que a média latino-americana $(1,9)$, embora quase a metade da média da OCDE $(4,8)$. Esse quadro permite conceber que qualquer controle indiscriminado dos custos em relação ao número de leitos hospitalares sobrecarregaria o SUS, que já está assoberbado por sua missão constitucional de cobertura universal de saúde. 
Tabela 2

Indicadores de oferta de serviços de saúde: profissionais de saúde e leitos hospitalares (por 1.000 habitantes)

\begin{tabular}{lccc}
\hline Países & Médicos & Enfermeiros & Leitos hospitalares \\
\hline Brasil & $2.1(\mathrm{a})$ & $0.5(\mathrm{~b})$ & $2.6(\mathrm{c})$ \\
Argentina & $3.2(\mathrm{~d})$ & $0.4(\mathrm{~d})$ & $4.1(\mathrm{a})$ \\
Chile & 1,1 & 0,6 & 2,5 \\
Colômbia & $1.3(\mathrm{c})$ & $0.5(\mathrm{c})$ & $1.2(\mathrm{~d})$ \\
México & 1,5 & 2,1 & 1 \\
Venezuela & $1.9(\mathrm{~b})$ & $0.8(\mathrm{a})$ & $0.9(\mathrm{e})$ \\
Média América Latina & 1,8 & 0,9 & 1,9 \\
\hline Alemanha & 3,4 & 9,7 & $8.6(\mathrm{~d})$ \\
Canadá & 2,1 & 10 & 4 \\
Holanda & 3,1 & 13,7 & $4.6(\mathrm{c})$ \\
Reino Unido & $2.3(\mathrm{c})$ & 7.9 (c) & 3,3 \\
Estados Unidos & 2,2 & 9,1 & 4 \\
Média OCDE & 2,6 & 10,1 & 4,8 \\
\hline
\end{tabular}

Fontes: World Health Statistics 2006; The World Health Report 2006; OECD Health Data 2005; Opas 2005 (Situacion de Salud en Las Americas: Indicadores básicos).

Notas: Elaboração Ipea; (a) 2000; (b) 2001; (d) 2004; (e) setor público.

A Tabela 3 mostra que o Brasil tem a maior taxa de mortalidade infantil, apesar do excelente desempenho do Programa de Saúde da Família (PSF), e a menor esperança de vida ao nascer, entre os países selecionados. Essas são, obviamente, medidas aproximadas do desempenho de um sistema de saúde, mas cabe ressaltar que 23 de cada mil bebês nascidos vivos no Brasil morrem antes de chegar a um ano de idade, taxa bem inferior aos países da OCDE e da própria América Latina (exceto México).

Tabela 3

Indicadores de saúde: mortalidade infantil e expectativa de vida ao nascer - 2004

\begin{tabular}{lcc}
\hline Países & $\begin{array}{c}\text { Mortalidade infantil } \\
\text { (por mil nascidos vivos) }\end{array}$ & $\begin{array}{c}\text { Esperança de vida ao nascer } \\
\text { (anos) }\end{array}$ \\
\hline Brasil(a) & 23 & 72 \\
Argentina & 16 & 75 \\
Chile & 8 & 78 \\
Colômbia & 18 & 73 \\
México & 23 & 75 \\
Venezuela & 16 & 74 \\
\hline Alemanha & 4 & 78 \\
Canadá & 5 & 80 \\
Holanda & 4 & 79 \\
Reino Unido & 5 & 79 \\
Estados Unidos & 6 & 77 \\
\hline
\end{tabular}

Fonte: World Health Statistics 2006; The World Health Report 2006; World Bank 2006 (World Development indicators). Notas: Elaboração Ipea; (a) PAHO-RIPSA 2008 (Indicadores Básicos Saúde Brasil: Conceitos e Aplicações). 
Não desconhecemos que o PSF contribuiu para reduzir as taxas de mortalidade infantil, um fator importante, embora não exclusivo, para o declínio da mortalidade infantil nos últimos anos (Macinko; Guanais; Souza, 2006).

Em suma, o Brasil gastou apenas US\$ 597 per capita com saúde, ou $7,6 \%$ de seu PIB em 2003, enquanto a média dos países selecionados da OCDE foi de US\$3.145, ou $10,8 \%$. Já a média do gasto dos países latinoamericanos selecionados foi de US\$ 622 ou $6,7 \%$ do PIB. Há 2,1 médicos para cada mil pessoas e uma proporção de leitos abaixo da taxa da OCDE, mas acima dos países latino-americanos. No que diz respeito às medidas de desempenho, o Brasil tem a maior taxa de mortalidade infantil, apesar do comportamento satisfatório do Programa de Saúde da Família, e a menor expectativa de vida no nascimento.

De forma geral, a reforma e as políticas de saúde introduzidas depois de 1988 parecem ter causado um impacto positivo na redução da pobreza e das desigualdades em saúde (Mesa-Lago, 2007). Contudo, o "momento político que permitiu (que elas) sobrevivessem à onda adversa de reformas neoliberais e, sobretudo, assegurassem que o gasto público em saúde recebesse uma parcela cada vez maior do PIB, começará a passar" (Cornwall e Shankland, 2008).

Nessa medida, para se garantir o mandato constitucional, é necessário criar uma fonte estável de financiamento para o SUS, que seja claramente progressiva (Ugá e Santos, 2007), revertendo o baixo nível de gasto público, a taxa reduzida de leitos públicos e os resultados inadequados em saúde.

\section{Considerações finais}

Como os formuladores de política podem lidar com a aspiração de produzir um sistema de saúde universal, em um contexto de restrição fiscal do Estado? Parece haver uma contradição entre o modelo redistributivo preconizado na Constituição brasileira e o nível de gasto público em saúde. Por exemplo, o National Health System inglês, modelo semelhante ao preconizado pela Constituição brasileira, apresenta um nível de gasto público em saúde que representa $85,7 \%$ do total de recursos (Maynard, 2005), enquanto o percentual do gasto público brasileiro é de $45,3 \%$.

Considerando esta restrição orçamentária, o Estado não tem sido capaz de responder às necessidades de cobertura, a um só tempo, impedindo que o SUS se fortaleça e deixando espaço para o crescimento do mercado de planos de saúde. De tal modo que o Brasil enfrentaria um sério problema na provisão pública da assistência à saúde, caso o financiamento do SUS fosse reduzido e o acesso ao sistema restringido. 
Os problemas de gestão do SUS decorrem também desta crise crônica de financiamento, porém o Ministério da Saúde e os secretários municipais e estaduais de saúde devem buscar incrementar a eficiência do SUS, alocando recursos com base nas necessidades de saúde da população, para promover a eqüidade e a melhoria da qualidade da atenção médica. Vale dizer, a partir da análise do sistema americano, Krugman e Wells (2006) sugerem que há pouca evidência de que “(...) o setor privado possa oferecer assistência à saúde mais eficiente do que a oferecida pelo governo", o que tornaria o papel do setor público para a organização do sistema de saúde ainda mais decisivo.

Como conclui a autora, "lamentavelmente, nesses longos anos, predomina a visão da saúde como fonte de despesa, afastando-a da idéia de direito social ou valor humano". Apesar desta aparente derrota ideológica, o clima de incerteza ao redor da proposta de reforma fiscal no futuro do orçamento da seguridade social (Silveira e Rangel, 2008; Delgado, 2008) poderá ser revertido com a introdução de uma nova fonte de financiamento estável e segura no longo prazo, tornando o SUS uma realidade mais próxima de nossos cidadãos e trabalhadores.

\section{Nota}

1 Pesquisador do Instituto de Pesquisa Econômica Aplicada (Ipea) e assessor da presidência da Agência Nacional de Saúde Suplementar (ANS). Doutor em Saúde Coletiva pela Universidade do Estado do Rio de Janeiro (Uerj). Pós-doutorado pela Yale University. $<$ carlos.octavio@ipea.gov.br>

Correspondência: Rua Conde de Irajá, 634, apto. 101, Botafogo, Rio de Janeiro, CEP 22271020 .

\section{Referências}

CASTRO, Jorge Abrahão de et al. Gasto social e política macroeconômica: trajetórias e tensões no período 1995-2005. Brasília: Ipea, 2008. (Texto para discussão n. ${ }^{\circ}$ 1324).

CONILL, Eleonor Minho. Sistemas comparados de saúde. In: CAMPOS, Gastão Wagner de Souza et al. (Ed.). Tratado de saúde cole- tiva. São Paulo; Rio de Janeiro: Hucitec e Editora Fiocruz, 2006. p. 563-613.

CORNWALL, Andrea; SHANKLAND, Alex. Engaging citizens: lessons from building Brazil's national health system. Social Science and Medicine, v. 66, n. 10, p. 2.1732.184, 2008. 
DELGADO, Guilherme Costa. Reforma tributária é o mais sorrateiro golpe na seguridade social. Correio da Cidadania, 2008. Disponível em: <www.correiocidadania .com.br/content/view/1949/109>. Acesso em: 30 jun. 2008.

DELFIM NETTO, Antônio. Saúde universal. Carta Capital, 2006. Disponível em: $<$ http://cms.cartacapital.com.br/carta/edico es/2006/12/423/saude-universal $>$. Acesso em: 30 jun. 2008 .

FAGIN, Claire. Cost effectiveness: nursing's value proves itself. The American Journal of Nursing, v. 90, n. 10, p. 16-30, 1990.

IPEA (Instituto de Pesquisa Econômica Aplicada). Boletim de Politicas Sociais, n. 15. Brasília: Ipea, mar. 2008.

KRUGMAN, Paul; WELLS, Robin. The health care crisis and what to do about it. The New York Review of Books, v. 53, n. 5, 2006. Disponível em: <www.nybooks.com/ articles/18802>. Acesso em: 4 dez. 2007.

MACINKO, James; GUANAIS, Frederico; SOUZA, Maria de Fátima Marinho de. Evaluation of the impact of the Family Health Program on infant mortality in Brazil, 1990-2002. Journal of Epidemiology and Community Health, v. 60, n. 1, p. 13-19, 2006

MARINHO, Alexandre. Um estudo sobre as filas para internações e para transplantes no Sistema Único de Saúde brasileiro. Rio de Janeiro: Ipea, 2004. (Texto para discussão n. $\left.{ }^{\circ} 1.055\right)$.

MARINHO, Alexandre; FAÇANHA, Luiz Otávio. Programas sociais: efetividade, eficiência e eficácia como dimensões operacionais da avaliação. Rio de Janeiro: Ipea, 2001 (Texto para discussão n. ${ }^{\circ}$ 787).

MARMOR, Theodore Richard. Comparing global health systems: lessons and caveats. In: WIENERS, W. W. (Ed.). Global health care markets: a comprehensive guide to regions, trends, and opportunities shaping the international health arena. San Franscisco: Jossey-Bass, 2001. p. 7-23.

MARMOR, Theodore Richard.; OKMA, Kieke.; LATHAM, Stephen. Values, institutions and health politics. In: WENDT, C., WOLF, C. (Eds). Soziologie der Gesundheit. Deutschland: VS Verlag, 2006. p. 383-405.

MAYNARD, Alan (Ed.) The public-private mix for health: plus ça change, plus c'est la même chose? United Kingdom: Nuffield Trust, 2005.

MARQUES, Rosa Maria; MENDES, Áquilas. Nogueira. Financiamento: a doença crônica da saúde pública brasileira. In: ENCONTRO NACIONAL DE ECONOMIA DA SAÚDE, 5. Anais, p. 213-238. Salvador: Encontro Nacional de Economia da Saúde, 5, 1999.

MESA-LAGO, Carmela. O sistema de saúde brasileiro: seu impacto na pobreza e na desigualdade. Nueva Sociedad (em português), 2007. Disponível em: <www.nuso.org/ esp_portugues/Mesa \%20Lago.pdf > . Acesso em: 30 jun. 2008.

MUSSI, Carlos; AFONSO, José Roberto. Como conciliar desenvolvimento econômico com bem-estar social? Nueva Sociedad, 215, maio-jun. 2008. Disponível em: $<$ www.nuso.org/upload/articulos/3527_2. pdf $>$. Acesso em: 30 jun. 2008.

OECD HEALTH DATA 2005. Statistics and Indicators for 30 Countries. Disponível em: $<$ http://www.oecdbookshop.org/oecd/disp lay.asp?k=5LHOLOPQZ5WK\&lang=en $>$. Acesso em: 21 out. 2008.

OCKÉ-REIS, Carlos Octávio. Sistemas de saúde comparados: gasto, acesso e desempenho. Rio de Janeiro: Ipea, 2006 (Lecture given at DIMAC n. 237, 11/08). Disponível em: <www.ipea.gov.br/sites/000/2/ destaque/seminario0237.pdf $>$. Acesso em: 9 jul. 2008.

ORGANIZACIÓN PANANAMERICANA DE LA SALUD. Situación de Salud en las Américas. Indicadores básicos 2005. Disponível 
em: <www.paho.org/spanish/dd/ais/IB-fol leto-2005.pdf>. Acesso em: 21 out. 2008.

PINHEIRO, Armando Castelar. Saúde: promessa social, desafio econômico. Valor [on line], 06/20/2008. Disponível em: $<$ www.valoronline.com.br/valoreconomico /285/primeirocaderno/opiniao/Saude+ promessa +social+desafio +econ $\%$ c $3 \%$ b4mico,,,58,4995209.html>. Acesso em: 30 jun. 2008.

REDE INTERAGENCIAL DE INFORMAÇÕES PARA SAÚDE (RIPSA). Indicadores básicos para saúde no Brasil 2008. Disponível em: $<$ http://tabnet.datasus.gov. br/cgi/idb2006/indicadores.pdf >. Acesso em: 21 out. 2008.

REMLER, Dahlia; BROWN, Lawrence; GLIED, Sherry. Market versus state in health care and health insurance: false dichotomy. In: NELSON, R. R. (ed.). The limits of market organization. New York: Russell Sage Foundation, 2005. p. 213-230.

SILVEIRA, Fernando Gaiger; RANGEL, Leonardo. Proposta de desoneração da contribuição sobre a folha. Valor [on line], 27 jun. 2008. Disponível em: <www.valor online.com.br/valoreconomico/285/p
rimeirocaderno/opiniao/Proposta + $\mathrm{de}+$ desoneracao $+\mathrm{da}+$ contribuicao + sobre + a + folha,, ,58,5008776.html?highlight $=$ \&ne wsid $=5008776 \&$ areaid $=58 \&$ editionid $=203$ 8>. Acesso em: 30 jun. 2008.

THE WORLD BANK GROUP. Governance in Brazil's Unified Health System (SUS). Raising the quality of public spending and resource management. Brazil: World Bank, 2007 (Report n. 36.601-BR).

World Development Indicators 2006. Disponível em: <http://devdata. worldbank.org/wdi2006/contents/Cover.ht m>. Acesso em: 21 out. 2008.

THE WORLD HEALTH REPORT 2006. Working Together for Health. Disponível em: $<$ http://www.who.int/whr/2006/en/>. Acesso em: 21 out. 2008.

UGÁ, Maria Alicia Dominguez; SANTOS, Isabela Soares. An analysis of equity in Brazilian health system financing. Health Affairs, v. 24, n. 4, p. 1.017-1.028, 2007.

WORLD HEALTH ORGANIZATION. World Health Statistics 2006. Disponível em: $<$ http://www.who.int/whosis/whostat2006 /en/index.html>. Acesso em: 21 out. 2008. 















